PROCEEDINGS OF THE

AMERICAN MATHEMATICAL SOCIETY

Volume 131, Number 10, Pages 3289-3295

S 0002-9939(03)07024-2

Article electronically published on April 1, 2003

\title{
CHARACTERIZATION OF THE MOD 3 COHOMOLOGY OF $E_{7}$
}

\author{
AKIRA KONO, JAMES P. LIN, AND OSAMU NISHIMURA \\ (Communicated by Paul Goerss) \\ Dedicated to Professor Shôrô Araki on his 70th birthday
}

\begin{abstract}
It is shown that the mod 3 cohomology of a homotopy associative mod $3 \mathrm{H}$-space which is rationally equivalent to the Lie group $E_{7}$ and which has integral 3-torsion is isomorphic to that of $E_{7}$ as a Hopf algebra over the mod 3 Steenrod algebra.
\end{abstract}

\section{INTRODUCTION}

The purpose of this paper is to show the following theorem.

Theorem 1. If a homotopy associative mod $3 H$-space $X$ is rationally equivalent to $E_{7}$ and has integral 3 -torsion, then $H^{*}\left(X ; \mathbb{F}_{3}\right)$ is isomorphic to $H^{*}\left(E_{7} ; \mathbb{F}_{3}\right)$ as a Hopf algebra over the mod 3 Steenrod algebra.

For the detail of $H^{*}\left(E_{7} ; \mathbb{F}_{3}\right)$, see Araki[2] and Kono-Mimura [1]. This theorem is known to be true if one replaces $E_{7}$ with $F_{4}$ or $E_{8}$ (see [14] and Kane [8], [9]).

The starting point of our proof of Theorem 1 is the loop space theorem for odd primes [14]. We recall some results and then we claim that $\wp^{3} \wp^{1} H^{3}\left(X ; \mathbb{F}_{3}\right) \neq 0$. Its proof, which is given in Section 3, is a combination of applications of an obstruction theory about lifting $a_{n}$-maps to $a_{n-1}$-maps in 2-stage Postnikov systems ([15]) with analyses of the $H_{*}\left(X ; \mathbb{F}_{3}\right)$-module Hopf algebra structures of $H_{*}\left(X ; \mathbb{F}_{3}\right)$ and $H_{*}\left(\Omega X ; \mathbb{F}_{3}\right)$ which are given by the adjoint actions ad: $X \times X \rightarrow X$ and Ad: $X \times$ $\Omega X \rightarrow \Omega X$, respectively. (For a Hopf algebra $A$, we say $B$ has an $A$-module Hopf algebra structure if $B$ has an $A$-module structure as well as a Hopf algebra structure such that the four structure maps of the Hopf algebra $B$, that is, the multiplication, the unit, the comultiplication, and the counit, are maps of $A$-modules where $A$ module structures of the ground ring and $B \otimes B$ are given as in Milnor-Moore 17]. See Kono-Kozima [10] and Hamanaka-Hara [5].) Then, we prove Theorem 1 by further analyses of the adjoint actions and by a factorization of $\wp^{3}$ through secondary operations ([16, [19]).

The most difficult part in determining the coalgebra structure of the mod 3 cohomology of $E_{7}$ is to determine the coproduct of $x_{35}$, the generator of degree 35 . (See 11].) Indeed, Mimura and the first-named author used the inclusion $E_{7} \hookrightarrow E_{8}$ to determine it, but no methods for determining it without using $E_{8}$ were known. Our proof of Theorem 1 gives such a method.

Received by the editors May 14, 2002 .

2000 Mathematics Subject Classification. Primary 57T05, 57T10, 57T25.

(C)2003 American Mathematical Society 


\section{ProOF}

We use the following notation. Given a vector space $V$ and its subset $S$, let $\langle S\rangle$ denote the subspace generated by $S$. The subscript of an element of a graded algebra designates the degree. Given a Hopf algebra $A$, let $\mathrm{P} A$ and $\mathrm{Q} A$ denote the primitives and the indecomposables respectively, and let $\bar{x} \in \mathrm{Q} A$ denote the class of an element $x$ of $A$. The coefficient for homology and cohomology is $\mathbb{F}_{3}$.

Let $X$ satisfy the hypothesis of Theorem 1 By [14, we have

$$
H^{*}(X)=\mathbb{F}_{3}\left[x_{8}\right] /\left(x_{8}^{3}\right) \otimes \bigwedge\left(x_{3}, x_{7}, x_{11}, x_{15}, x_{19}, x_{27}, x_{35}\right)
$$

as an algebra and we have $x_{7}=\wp^{1} x_{3}$ and $x_{8}=\beta x_{7}$. Let $B=\mathbb{F}_{3}\left[x_{8}\right] /\left(x_{8}^{3}\right)$ and $R=$ $\langle 1\rangle \oplus\left\{x \in H^{*}(X)\left|\bar{\Delta} x \in B \otimes H^{*}(X),\right| x \mid>0\right\}$ where $\bar{\Delta}$ is the reduced coproduct of $H^{*}(X)$. By Baum-Browder 3 and Kane [9, $R$ is a coalgebra over $\mathcal{A}(3)$, the mod 3 Steenrod algebra. Further $R=B$ in even degrees and $R$ is (naturally) isomorphic to $\mathrm{Q} H^{*}(X)$ in odd degrees. Hence we can choose $x_{j}$ for $j=11,15,19,27,35$ so that $R=\left\langle x_{j}\right| j$ is odd $\rangle$ in odd degrees. Let $a_{j} \in \mathrm{P} H_{*}(X)$ be the dual element of $\bar{x}_{j} \in \mathrm{Q} H^{*}(X)$. By Kane [8], we may assume that $a_{11}=a_{8} * a_{3}=a_{15} \wp^{1}$ and that $a_{15}=a_{8} * a_{7}$ where $*$ indicates the adjoint action. (Note that $a * b=[a, b]$ if $a$ is primitive. See Kono-Kozima [10] and Hamanaka-Hara [5].) Moreover, we can choose $x_{19}$ to satisfy

Proposition 2. $x_{19}=\wp^{3} x_{7}$.

The proof is given in Section 3. By Proposition 2, we have $\left(a_{8} * a_{19}\right) \wp^{3}=a_{8} * a_{7}=$ $a_{15}$ and hence we may assume that $a_{27}=a_{8} * a_{19}$ and that $a_{27} \wp^{3}=a_{15}$.

Let $\sigma: H_{*}(\Omega X) \rightarrow \mathrm{P} H_{*}(X)$ be the homology suspension. By Clark [4], Kane [7], and Kraines [13, we have

$$
H_{*}(\Omega X)=\mathbb{F}_{3}\left[t_{2}, t_{6}, t_{10}, t_{14}, t_{18}, t_{22}, t_{26}, t_{34}\right] /\left(t_{2}^{3}\right)
$$

as an algebra where $\sigma\left(t_{j}\right)=a_{j+1}$ for $j \neq 22$. Since $H_{*}(\Omega X)$ is bicommutative, we may assume that $t_{j} \in \mathrm{P} H_{*}(\Omega X)$ for $j \neq 6,18$. Moreover, we can easily see that $\bar{\phi}\left(t_{6}\right)=-t_{2}^{2} \otimes t_{2}-t_{2} \otimes t_{2}^{2}$ where $\bar{\phi}$ is the reduced coproduct of $H_{*}(\Omega X)$.

The relations $a_{8} * a_{3}=a_{11}$ and $a_{8} * a_{7}=a_{15}$ are converted into $a_{8} * t_{2}=t_{10}$ and $a_{8} * t_{6}=t_{14}-t_{10} t_{2}^{2}$, respectively. (See Hamanaka-Hara [5].) Put $v=a_{8} * t_{10} \in$ $\mathrm{P} H_{*}(\Omega X)$. Since $a_{8}^{3}=0$, we have $a_{8} * v=a_{8}^{2} * t_{10}=a_{8}^{3} * t_{2}=0$ and

$$
0=a_{8}^{3} * t_{6}=a_{8}^{2} *\left(t_{14}-t_{10} t_{2}^{2}\right)=\cdots=a_{8}^{2} * t_{14}+t_{10}^{3} .
$$

Hence we have $a_{8}^{2} * t_{14}=-t_{10}^{3} \neq 0$.

The relation $a_{27} \wp^{3}=a_{15}$ is converted into $t_{26} \wp^{3}=t_{14}$. Then, we have

$$
\left(a_{8}^{2} * t_{26}\right) \wp^{3}=a_{8}^{2} * t_{14} \neq 0
$$

and hence we have $a_{8} * t_{26} \neq 0$. Thus we may assume that $t_{34}=a_{8} * t_{26}$. This is converted into $a_{35}=a_{8} * a_{27}$. Then, we can easily see that $H^{*}(X)$ is isomorphic to $H^{*}\left(E_{7}\right)$ as a Hopf algebra over $\mathbb{F}_{3}$. (Recall that $H_{*}(X)$ is the universal enveloping Hopf algebra of the restricted Lie algebra $\mathrm{P}_{*}(X)$ over $\mathcal{A}(3)$. See Milnor-Moore [17] and Kane [9.)

For the Steenrod module structure, we are left to show that $a_{19} \wp^{1}= \pm a_{15}$. Note that $\wp^{3} x_{15}=x_{27}$. Let $X\langle 3\rangle$ be the 3 -connective cover of $X$ and $g: X\langle 3\rangle \rightarrow X$ 
the covering projection. By the Serre spectral sequence, we have $H^{*}(X\langle 3\rangle)=$ $\bigwedge\left(\hat{x}_{11}, \hat{x}_{15}, \hat{x}_{27}, \hat{y}_{19}, \hat{y}_{23}\right)$ for $*<35$ where $\hat{x}_{j}=g^{*}\left(x_{j}\right)$ and $\hat{y}_{j} \notin \operatorname{Im} g^{*}$. Note that $\beta \hat{x}_{15}=\wp^{1} \hat{x}_{15}=0$ and that $\wp^{3} \hat{x}_{15}=\hat{x}_{27}$. According to Liulevicius [16] or Shimada-Yamanoshita [19], the primary operation $\wp^{3} \hat{x}_{15}=\hat{x}_{27}$ decomposes into $\Phi \hat{x}_{15}=\hat{y}_{23}$ and $\wp^{1} \hat{y}_{23}= \pm \hat{x}_{27}$ where $\Phi$ is a secondary operation. Then, we can easily show that $t_{26} \wp^{1}= \pm t_{22}$ by the homotopy fiberings $\Omega(X\langle 3\rangle) \rightarrow * \rightarrow X\langle 3\rangle$ and $S^{1} \rightarrow \Omega(X\langle 3\rangle) \stackrel{\Omega g}{\rightarrow} \Omega X$.

The relation $a_{8} * a_{19}=a_{27}$ is converted into $a_{8} * \bar{t}_{18}=\bar{t}_{26}$. (Note that $\mathrm{Q} H_{*}(\Omega X)$ has an $H_{*}(X)$-module structure which is induced from the $H_{*}(X)$-module Hopf algebra structure of $H_{*}(\Omega X)$ which is defined by the adjoint action. We also use the symbol $*$ to denote this structure.) Hence we have

$$
a_{8} *\left(\bar{t}_{18 \wp^{1}}\right)=\left(a_{8} * \bar{t}_{18}\right) \wp^{1}=\bar{t}_{26} \wp^{1}= \pm \bar{t}_{22} \neq 0 .
$$

Thus we have $\bar{t}_{18 \wp^{1}} \neq 0$ and hence $\bar{t}_{18 \wp^{1}}= \pm \bar{t}_{14}$. This is converted into $a_{19} \wp^{1}=$ $\pm a_{15}$.

\section{Proof of Proposition 2}

Note that $\wp^{1} x_{15} \in R^{19}=\left\langle x_{19}\right\rangle$.

3.1. Case (i). First, assume that $\wp^{3} x_{7}=0$ and that $\wp^{1} x_{15} \neq 0$. We will show a contradiction. We may assume that $\wp^{1} x_{15}=x_{19}$ and then we have $a_{19} \wp^{1}=a_{15}$.

By the same argument as that in Section 2, we know that $H_{*}(\Omega X)$ is as in (2.1) where $\sigma\left(t_{j}\right)=a_{j+1}$ for $j \neq 22, t_{j} \in \mathrm{P} H_{*}(\Omega X)$ for $j \neq 6,18$, and $\bar{\phi}\left(t_{6}\right)=-t_{2}^{2} \otimes t_{2}-$ $t_{2} \otimes t_{2}^{2}$. Also we have $a_{8} * t_{2}=t_{10}, a_{8} * t_{6}=t_{14}-t_{10} t_{2}^{2}$, and $a_{8}^{2} * t_{14}=-t_{10}^{3} \neq 0$. Thus we have $0 \neq a_{8} * t_{14} \in \mathrm{P} H_{22}(\Omega X)$ and we may assume that $a_{8} * t_{14}=t_{22}$.

The relation $a_{19} \wp^{1}=a_{15}$ is converted into $\bar{t}_{18} \wp^{1}=\bar{t}_{14}$. It follows that

$$
\left(a_{8} * \bar{t}_{18}\right) \wp^{1}=a_{8} *\left(\bar{t}_{18} \wp^{1}\right)=a_{8} * \bar{t}_{14}=\bar{t}_{22} .
$$

Thus, we have $a_{8} * \bar{t}_{18} \neq 0$ and hence we may assume that $\bar{t}_{26}=a_{8} * \bar{t}_{18}$.

Here we again consider $g: X\langle 3\rangle \rightarrow X$. The Serre spectral sequence implies that

$$
H^{*}(X\langle 3\rangle)=\bigwedge\left(\hat{x}_{11}, \hat{x}_{15}, \hat{x}_{19}, \hat{x}_{27}, \hat{x}_{35}\right) \otimes \mathbb{F}_{3}\left[\hat{y}_{18}\right] \otimes \bigwedge\left(\hat{y}_{19}, \hat{y}_{23}\right)
$$

where $\hat{x}_{i}=g^{*}\left(x_{i}\right)$. Further, by the Serre spectral sequence for the homotopy fibering $X\langle 3\rangle \stackrel{g}{\rightarrow} X \rightarrow K(\mathbb{Z}, 3)$, we may assume that $\beta \hat{y}_{18}=\hat{y}_{19}$ and that $\wp^{1} \hat{y}_{19}=$ $\hat{y}_{23}$. By the homotopy fiberings $\Omega(X\langle 3\rangle) \rightarrow * \rightarrow X\langle 3\rangle$ and $S^{1} \rightarrow \Omega(X\langle 3\rangle) \stackrel{\Omega g}{\rightarrow} \Omega X$, we can easily show that (3.1) implies $\wp^{1} \hat{y}_{23}= \pm \hat{x}_{27}$. It follows that

$$
\pm \hat{x}_{27}=\wp^{1} \wp^{1} \beta \hat{y}_{18}=\left(-\beta \wp^{1} \wp^{1}-\wp^{1} \beta \wp^{1}\right) \hat{y}_{18}
$$

by the Adem relation. Since $\wp^{1} \hat{y}_{18} \in H^{22}(X\langle 3\rangle)=0$, this is a contradiction.

3.2. Case (ii). Now, assume that $\wp^{3} x_{7}=0$ and that $\wp^{1} x_{15}=0$. We can show a contradiction and thus, we have Proposition 2

First, note that we have $\bar{\Delta} x_{11}=x_{8} \otimes x_{3}, \bar{\Delta} x_{15}=x_{8} \otimes x_{7}$, and $\wp^{1} x_{11}=x_{15}$. Moreover, since $R^{23}=R^{31}=0$, we have $\wp^{1} x_{19}=\wp^{3} x_{19}=0$. We may put

$$
\bar{\Delta} x_{19}=a x_{8}^{2} \otimes x_{3}+b x_{8} \otimes x_{11} \quad a, b \in \mathbb{F}_{3} .
$$

Applying $\wp^{1}$, we have

$$
0=\bar{\Delta}_{\wp}{ }^{1} x_{19}=a x_{8}^{2} \otimes x_{7}+b x_{8} \otimes x_{15} .
$$


Hence $a=b=0$. So $x_{19}$ is primitive. Similarly, we have $\wp^{1} x_{27}=0$ and we can show that $\bar{\Delta} x_{27} \in\left\langle x_{8} \otimes x_{19}\right\rangle$.

Let $i_{k}$ denote the fundamental class of $K\left(\mathbb{F}_{3}, k\right)$ and put $K\left(\mathbb{F}_{3}, k, l\right)=K\left(\mathbb{F}_{3}, k\right) \times$ $K\left(\mathbb{F}_{3}, l\right)$. Let $w: K\left(\mathbb{F}_{3}, 19\right) \rightarrow K\left(\mathbb{F}_{3}, 23,31\right)$ be defined by $w^{*}\left(i_{23}\right)=\wp^{1} i_{19}$ and $w^{*}\left(i_{31}\right)=\wp^{3} i_{19}$. Let $\alpha: K\left(\mathbb{F}_{3}, 23,31\right) \rightarrow K\left(\mathbb{F}_{3}, 23,47\right)$ be defined by $\alpha^{*}\left(i_{23}\right)=i_{23}$ and $\alpha^{*}\left(i_{47}\right)=\wp^{4} i_{31}$ and put $w_{1}=\alpha \circ w$. Let $E$ and $E_{1}$ be the fibres of $w$ and $w_{1}$, respectively. Then we have a homotopy commutative diagram

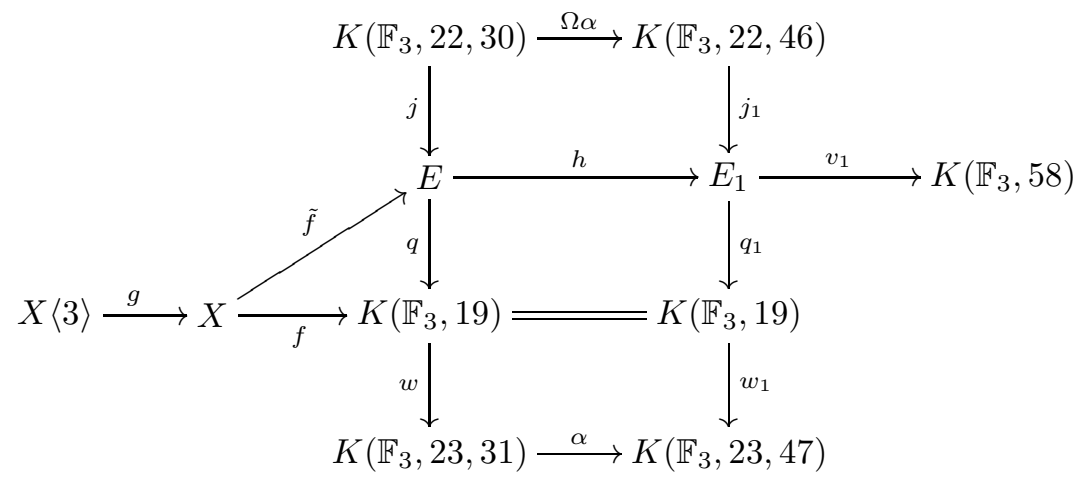

where the vertical sequences are homotopy fibre sequences, $f^{*}\left(i_{19}\right)=x_{19}$, and $\tilde{f}$ is a lift of $f$. There is an Adem relation

$$
\wp^{10}=\wp^{9} \wp^{1}+\wp^{3} \wp^{4} \wp^{3} .
$$

Hence there is an $H$-map $v_{1}: E_{1} \rightarrow K\left(\mathrm{~F}_{3}, 58\right)$ with $\left(v_{1} \circ j_{1}\right)^{*}\left(i_{58}\right)=\wp^{9} i_{22}+\wp^{3} i_{46}$. By Hemmi [6], we have $\left[a_{3}\left(v_{1}\right)\right]=u_{1} \otimes u_{1} \otimes u_{1} \in H^{57}\left(E_{1} \wedge E_{1} \wedge E_{1}\right)$ where $a_{3}\left(v_{1}\right)$ is the $a_{3}$-deviation of $v_{1}$ and $u_{1}=q_{1}^{*}\left(i_{19}\right)$.

Step 1. We will prove that $f \circ g$ is an $a_{3}$-map and that $\tilde{f}$ can be chosen so that $h \circ \tilde{f} \circ g$ is an $H$-map. Note that $\left[a_{3}(f)\right] \in H^{18}(X \wedge X \wedge X)$ must be a sum of terms involving $x_{3}, x_{7}, x_{8}$. Put $A=\left\langle x_{3}, x_{7}, x_{8}\right\rangle$, which is a subspace of $H^{*}(X)$. Note that $A$ is invariant under $\mathcal{A}(3)$ and that $g^{*}(A)=0$. So $a_{3}(f \circ g) \simeq a_{3}(f) \circ(g \wedge g \wedge g) \simeq *$ and thus $f \circ g$ is an $a_{3}$-map.

Let $\gamma: X \wedge X \rightarrow K\left(\mathbb{F}_{3}, 22,30\right)$ be a lift of $D_{\tilde{f}}: X \wedge X \rightarrow E$ where $D_{\tilde{f}}$ is the $H$-deviation of $\tilde{f}$. By Zabrodsky [20], $a_{3}(w \circ f) \simeq(\Omega w) \circ a_{3}(f)$ represents $(\bar{\Delta} \otimes 1-1 \otimes \bar{\Delta})[\gamma]$ under identifications

$$
\left[X \wedge X, K\left(\mathbb{F}_{3}, 22,30\right)\right]=H^{22}(X \wedge X) \oplus H^{30}(X \wedge X)
$$

and

$$
\left[X \wedge X \wedge X, \Omega K\left(\mathbb{F}_{3}, 23,31\right)\right]=H^{22}(X \wedge X \wedge X) \oplus H^{30}(X \wedge X \wedge X) .
$$

Hence

$$
\begin{aligned}
\wp^{1}\left[a_{3}(f)\right] & =(\bar{\Delta} \otimes 1-1 \otimes \bar{\Delta}) \gamma^{*}\left(i_{22}\right), \\
\wp^{3}\left[a_{3}(f)\right] & =(\bar{\Delta} \otimes 1-1 \otimes \bar{\Delta}) \gamma^{*}\left(i_{30}\right) .
\end{aligned}
$$


We can check that $\wp^{1}\left[a_{3}(f)\right]$ must be a sum of terms involving $x_{7}, x_{7}, x_{8}$ and that $\wp^{3}\left[a_{3}(f)\right]=0$. We can find an element

$$
\xi \in\left\langle x_{7} \otimes x_{7} x_{8}, x_{7} x_{8} \otimes x_{7}, x_{7} \otimes x_{15}, x_{15} \otimes x_{7}\right\rangle
$$

such that $(\bar{\Delta} \otimes 1-1 \otimes \bar{\Delta})(\xi)=\wp^{1}\left[a_{3}(f)\right]$. Thus $\gamma^{*}\left(i_{22}\right)-\xi$ and $\gamma^{*}\left(i_{30}\right)$ lie in $\operatorname{Ker}(\bar{\Delta} \otimes 1-1 \otimes \bar{\Delta})$.

Here we think about $\operatorname{Cotor}_{H^{*}(X)}^{2, j}\left(\mathbb{F}_{3}, \mathbb{F}_{3}\right)$ for $j=22,30$. Recall that $\bar{\Delta} x_{27} \in$ $\left\langle x_{8} \otimes x_{19}\right\rangle$.

If $x_{27}$ is primitive, we have $H^{*}(X) \cong C \otimes C^{\prime} \otimes C^{\prime \prime}$ for $*<35$ as a Hopf algebra over $\mathbb{F}_{3}$ where $C, C^{\prime}$, and $C^{\prime \prime}$ are Hopf algebras over $\mathbb{F}_{3}$ which are isomorphic to $H^{*}\left(F_{4}\right), H^{*}\left(S^{19}\right)$, and $H^{*}\left(S^{27}\right)$, respectively. We know the module structure of $\operatorname{Cotor}_{C}^{*, *}\left(\mathbb{F}_{3}, \mathbb{F}_{3}\right)$ by Kono-Mimura-Shimada 12 . Then we can easily show that

$$
\begin{aligned}
& \operatorname{Cotor}_{H^{*}(X)}^{2,22}\left(\mathbb{F}_{3}, \mathbb{F}_{3}\right)=\left\langle\left\{x_{3} \otimes x_{19}\right\}\right\rangle, \\
& \operatorname{Cotor}_{H^{*}(X)}^{2,30}\left(\mathbb{F}_{3}, \mathbb{F}_{3}\right)=\left\langle\left\{x_{3} \otimes x_{27}\right\}\right\rangle .
\end{aligned}
$$

If $x_{27}$ is not primitive, then we have $H^{*}(X) \cong H^{*}\left(E_{7}\right)$ for $*<35$ as a Hopf algebra over $\mathbb{F}_{3}$. We know the module structure of $\operatorname{Cotor}_{H^{*}\left(E_{7}\right)}^{* *}\left(\mathbb{F}_{3}, \mathbb{F}_{3}\right)$ by MimuraSambe [18]. Then we can easily show that $\operatorname{Cotor}_{H^{*}(X)}^{2,22}\left(\mathbb{F}_{3}, \mathbb{F}_{3}\right)$ is as in $(3.3)$ and $\operatorname{Cotor}_{H^{*}(X)}^{2,30}\left(\mathbb{F}_{3}, \mathbb{F}_{3}\right)=\langle\{\eta\}\rangle$ where

$$
\eta=x_{3} \otimes x_{27}-x_{19} \otimes x_{11}+x_{3} x_{8} \otimes x_{19}+x_{8} \otimes x_{3} x_{19}-x_{8} x_{19} \otimes x_{3} .
$$

Thus we may put $\gamma^{*}\left(i_{22}\right)$ as $\gamma^{*}\left(i_{22}\right)=\xi+\varepsilon x_{3} \otimes x_{19}+l$ and $\gamma^{*}\left(i_{30}\right)$ either as $\gamma^{*}\left(i_{30}\right)=\varepsilon^{\prime} x_{3} \otimes x_{27}+l^{\prime}$ or as $\gamma^{*}\left(i_{30}\right)=\varepsilon^{\prime} \eta+l^{\prime}$ where $\varepsilon, \varepsilon^{\prime} \in \mathbb{F}_{3}$ and $l, l^{\prime} \in \operatorname{Im} \bar{\Delta}$. As explained in [15], we can alter $\gamma$ so that $l=l^{\prime}=0$. Note that $\wp^{4}\left(x_{19} \otimes x_{11}\right)=$ $\wp^{4}\left(x_{3} x_{8} \otimes x_{19}\right)=0$ and hence $\wp^{4} \eta \in A \otimes H^{*}(X)+H^{*}(X) \otimes A$. (In fact, $\wp^{4} \eta=0$. Recall that $R$ is invariant under $\mathcal{A}(3)$.) It follows that $((\Omega \alpha) \circ \gamma)^{*}\left(i_{22}\right)=\gamma^{*}\left(i_{22}\right)$ and $((\Omega \alpha) \circ \gamma)^{*}\left(i_{46}\right)=\wp^{4} \gamma^{*}\left(i_{30}\right)$ lie in $A \otimes H^{*}(X)+H^{*}(X) \otimes A$. Hence $D_{h \circ \tilde{f} \circ g} \simeq$ $h \circ D_{\tilde{f}} \circ(g \wedge g) \simeq h \circ j \circ \gamma \circ(g \wedge g) \simeq j_{1} \circ(\Omega \alpha) \circ \gamma \circ(g \wedge g) \simeq *$ and thus $h \circ \tilde{f} \circ g$ is an $H$-map. This proves Step 1.

Step 2. We will show a contradiction. We know that $H^{*}(X\langle 3\rangle)$ is as in (3.2). By Step $1, h \circ \tilde{f} \circ g$ is an $H$-map, so $\left(v_{1} \circ h \circ \tilde{f} \circ g\right)^{*}\left(i_{58}\right) \in \mathrm{P} H^{58}(X\langle 3\rangle)=0$. It follows that

$$
\begin{aligned}
{\left[a_{3}\left(v_{1} \circ h \circ \tilde{f} \circ g\right)\right]=} & {\left[a_{3}\left(v_{1}\right) \circ((h \circ \tilde{f} \circ g) \wedge(h \circ \tilde{f} \circ g) \wedge(h \circ \tilde{f} \circ g))\right] } \\
& \quad+\left[\left(\Omega v_{1}\right) \circ a_{3}(h \circ \tilde{f} \circ g)\right] \\
= & \hat{x}_{19} \otimes \hat{x}_{19} \otimes \hat{x}_{19}+\wp^{9} y+\wp^{3} y^{\prime} \\
= & (\bar{\Delta} \otimes 1-1 \otimes \bar{\Delta})(z) \\
\in & H^{57}(X\langle 3\rangle \wedge X\langle 3\rangle \wedge X\langle 3\rangle)
\end{aligned}
$$

for some $y, y^{\prime} \in H^{*}(X\langle 3\rangle \wedge X\langle 3\rangle \wedge X\langle 3\rangle)$ and $z \in H^{*}(X\langle 3\rangle \wedge X\langle 3\rangle)$ since $f \circ g$ is an $a_{3}$-map. Checking degrees, we have $y=0$. Since we assume that $\wp^{1} x_{15}=$ 0 , we have $\wp^{1} \hat{x}_{15}=0$. Let $H^{19,19,19}$ be the summand of $H^{57}(X\langle 3\rangle \wedge X\langle 3\rangle \wedge$ $X\langle 3\rangle)$ which corresponds to $H^{19}(X\langle 3\rangle) \otimes H^{19}(X\langle 3\rangle) \otimes H^{19}(X\langle 3\rangle)$, and let $\pi$ : $H^{57}(X\langle 3\rangle \wedge X\langle 3\rangle \wedge X\langle 3\rangle) \rightarrow H^{19,19,19}$ be the natural projection. Then, we can easily check that $\pi\left(\wp^{3} H^{45}(X\langle 3\rangle \wedge X\langle 3\rangle \wedge X\langle 3\rangle)\right)=0$. Accordingly, (3.4) implies that

$$
\hat{x}_{19} \otimes \hat{x}_{19} \otimes \hat{x}_{19}=\pi(\bar{\Delta} \otimes 1-1 \otimes \bar{\Delta})(z) .
$$


If $t \in \mathrm{P} H_{19}(X\langle 3\rangle)$ with $\left\langle t, \hat{x}_{19}\right\rangle \neq 0$, then

$$
\begin{aligned}
0 & \neq\left\langle t \otimes t \otimes t, \hat{x}_{19} \otimes \hat{x}_{19} \otimes \hat{x}_{19}\right\rangle \\
& =\langle t \otimes t \otimes t, \pi(\bar{\Delta} \otimes 1-1 \otimes \bar{\Delta})(z)\rangle \\
& =\langle t \otimes t \otimes t,(\bar{\Delta} \otimes 1-1 \otimes \bar{\Delta})(z)\rangle \\
& =\left\langle t^{2} \otimes t-t \otimes t^{2}, z\right\rangle .
\end{aligned}
$$

So $t^{2} \in \mathrm{P} H_{38}(X\langle 3\rangle)$ is nonzero. This is a contradiction.

\section{ACKNOWLEDGEMENT}

The third-named author was partially supported by JSPS Research Fellowships for Young Scientists. Part of this work was done during his visit to Aberdeen. The visit was supported by the British Council Grants for JSPS Fellows. He thanks Professor John Hubbuck, and also the Department of Mathematical Sciences, University of Aberdeen, and the British Council.

\section{REFERENCES}

[1] S. Araki : On the non-commutativity of Pontrjagin rings mod 3 of some compact exceptional groups. Nagoya Math. J. 17 (1960) 225-260. MR 25:3550a; correction MR 25:3550b

[2] S. Araki : Differential Hopf algebras and the cohomology mod 3 of the compact exceptional groups $E_{7}$ and $E_{8}$. Ann. of Math. (2) 73 (1961) 404-436. MR 23:A1372

[3] P. F. Baum-W. Browder : The cohomology of quotients of classical groups. Topology 3 (1965) 305-336. MR 32:6490

[4] A. Clark : Homotopy commutativity and the Moore spectral sequence. Pacific J. Math. 15 (1965) 65-74. MR 31:1679

[5] H. Hamanaka-S. Hara : The mod 3 homology of the space of loops on the exceptional Lie groups and the adjoint action. J. Math. Kyoto Univ. 37 (1997), no. 3, 441-453. MR 98m:57047

[6] Y. Hemmi : Mod 3 homotopy associative $H$-spaces which are products of spheres. Hiroshima Math. 18 (1988) 227-243. MR 89i:55005

[7] R. Kane : On loop spaces without $p$ torsion. Pacific J. Math. 60 (1975), no. 1, 189-201. MR 52:4277

[8] R. Kane : Torsion in homotopy associative $H$-spaces. Illinois J. Math. 20 (1976), no. 3, 476-485. MR 54:6131

[9] R. Kane: The homology algebra of finite $H$-spaces. J. Pure Appl. Algebra 41 (1986), no. 2-3, 213-232. MR 87k:55011

[10] A. Kono-K. Kozima : The adjoint action of a Lie group on the space of loops. J. Math. Soc. Japan 45 (1993), no. 3, 495-510. MR 94h:57053

[11] A. Kono-M. Mimura : Cohomology operations and the Hopf algebra structures of the compact, exceptional Lie groups $E_{7}$ and $E_{8}$. Proc. London Math. Soc. (3) 35 (1977), no. 2, 345-358. MR 56:13243

[12] A. Kono-M. Mimura-N. Shimada : Cohomology of classifying spaces of certain associative $H$-spaces. J. Math. Kyoto Univ. 15-3 (1975) 607-617. MR 52:9262

[13] D. Kraines: The kernel of the loop suspension map. Illinois J. Math. 21 (1977), no. 1, 91-108. MR 54:11334

[14] J. P. Lin : Torsion in $H$-spaces II. Ann. of Math. (2) 107 (1978), no. 1, 41-88. MR 58:7619

[15] J. P. Lin : $H$-spaces with finiteness conditions. Handbook of algebraic topology, NorthHolland, Amsterdam (1995) 1095-1141. MR 97c:55017

[16] A. Liulevicius : The factorization of cyclic reduced powers by secondary cohomology operations. Proc. Nat. Acad. Sci. U.S.A. 46 (1960) 978-981. MR 24:A2383

[17] J. Milnor-C. Moore : On the structure of Hopf algebras. Ann. of Math. (2) 81 (1965) 211-264. MR 30:4259

[18] M. Mimura-Y. Sambe : On the cohomology mod $p$ of the classifying spaces of the exceptional Lie groups, I. J. Math. Kyoto Univ. 19-3 (1979) 553-581. MR 81c:55025 
[19] N. Shimada-T. Yamanoshita : On triviality of the mod $p$ Hopf invariant. Japan J. Math. 31 (1961) 1-25. MR 26:5569

[20] A. Zabrodsky : Cohomology operations and homotopy commutative $H$-spaces. Springer Lecture Notes in Math, vol. 168 (1970) 308-317. MR 42:6815

Department of Mathematics, Kyoto University, Kyoto, 606-8502, Japan

E-mail address: kono@kusm.kyoto-u.ac.jp

Department of Mathematics, University of California, San Diego, La Jolla, CaliFORNIA 92093-0112

E-mail address: jimlin@euclid.ucsd.edu

Department of Mathematics, Kyoto University, Kyoto, 606-8502, Japan

E-mail address: osamu@kusm.kyoto-u.ac.jp 